\title{
A New System for Testing the Static Performance of MCP-PMT
}

\author{
Jiawei Shen , Jia Cao , Yiting Guo and Yunsheng Qian* \\ School of Electronic and Optical Engineering \\ Nanjing University of Science and Technology \\ Nanjing 210094, Jiangsu, China \\ *yshqian@mail.njust.edu.cn
}

Keywords: MCP-PMT, gain, quantum efficiency, power spectral density

\begin{abstract}
The microchannel-plate photomultiplier tube (MCP-PMT) is the key component in the Daya Bay reactor neutrino experiment $I I$. However, there are few approaches to evaluate its properties. Based on the situation, a testing system and a series of experiments to assess the static performance of MCP-PMT have been developed. The MCP-PMT with 8 inch diameter applied with high voltage is illuminated by appropriate incident light. The cathode and anode current are sampled by capture card to calculate static performance of MCP-PMT. The power spectral density of the anode output current is investigated with FFT. After the experiments are conducted, the system is qualified to test and analyze the static performance of studied MCP-PMT.
\end{abstract}

\section{Introduction}

Microchannel plate(MCP) is a device of arrays of small pores oriented in parallel to each other acting as electron multipliers[1]. The MCP-PMT has several advantages that make it a promising photon detector in high energy physics. High amplification, excellent time resolution, good spatial resolution and compact size result in an increasing interest to the applications of MCP-PMT in practice[2]. As shown in Fig. 1 and Fig.2, the MCP-PMT with 8 inch diameter mainly consists of transmission photocathode, reflection photocathode, anode, focusing electrode and two sets of double MCPs. Photoelectrons emitted from round photocathode will be collected by the focusing electrode. Then the electrons are multiplied through MCP1 and MCP2. The amplified current is measured at the anode. The transmission and reflection photocathode are coated on the spherical envelope to form $4 \pi$ viewing angle photocathode[3].

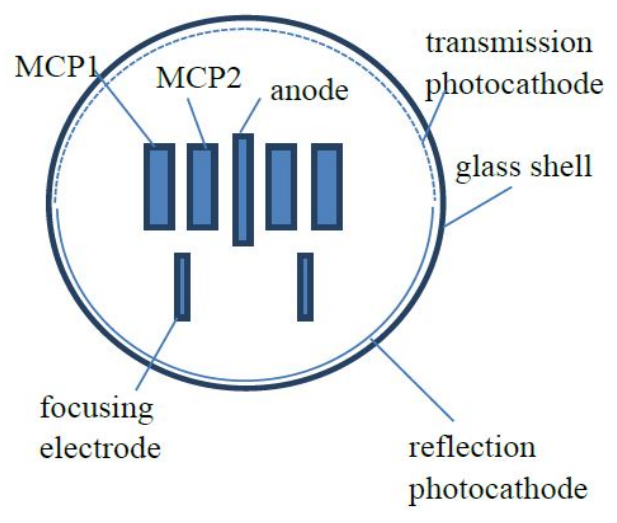

Fig. 1: The structure of MCP-PMT

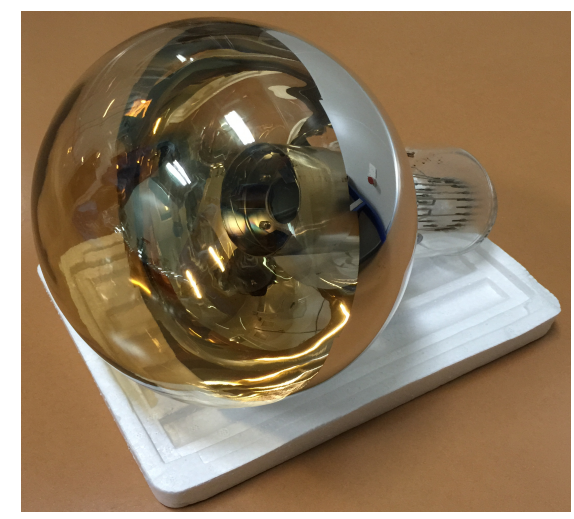

Fig. 2: The MCP-PMT with 8 inch diameter

\section{Parameters}

The performance evaluation of MCP-PMT includes the parameters: gain, photocathode sensitivity, anode sensitivity, spectrum sensitivity, quantum efficiency and noise power spectrum.

Gain. The gain (G) of MCP-PMT is defined as the ratio of anode current $I_{A}$ and cathode current $I_{K}$. According to the definition of cathode sensitivity $R_{K}$ and anode sensitivity $R_{A}, G=R_{A} / R_{K}$ can be deduced. 
The gain of single MCP is influenced by the voltage between anode and cathode, material to fabricate the MCP, structure of MCP and the incident electrons. Because it is impractical to measure the secondary electron emission, $G=R_{A} / R_{K}$ is actually used to calculate the gain.

Gain of the double MCP-PMT also changes with the distance and channel bias angle between the two MCPs. To achieve the right combination of gain and transit-time-spread(TTS), the distance between two MCPs should be $20-30 \mu m$, while the channel bias angle is always between $90^{\circ}$ and $180^{\circ}[4]$.

Spectrum Sensitivity and Quantum Efficiency(QE). The cathode spectrum sensitivity is defined as the ratio of cathode current $I_{K}$ and radiant flux $E$. The cathode current can be calculated with Eq.1, in which $E \lambda /(h c)$ denotes the number of incident photons. From Eq.1, the Eq.2 can be deduced to calculate $Q E$. If the unit of $\lambda$ is $\mu m$, the unit of $R_{K \lambda}$ should be $A / W[5]$.

$$
\begin{aligned}
& I_{K}=\frac{E \lambda e \eta(\lambda)}{h c} . \\
& \eta(\lambda)=\frac{1.24 I_{K}}{\lambda E}=\frac{1.24 R_{K \lambda}}{\lambda} .
\end{aligned}
$$

Noise Power Spectrum. The power spectrum estimation is an important method for spectrum analysis of MCP-PMT's noise performance. The power spectral density(PSD) describes how the power of a signal or time series is distributed over the different frequencies. For a stationary random process, the power spectral density $S_{x x}(\omega)$ and the autocorrelation function $\gamma(\tau)=\langle X(t) X(t+\tau)\rangle$ of the signal should be a Fourier transform pair[6]. In the experiment, $X(t)$ denotes the anode output current.

The power spectral density can actually be computed with the Eq.3. On the other hand, the method that approaches the power spectral density through the autocorrelation function will restrain the thermal noise and provide accurate results.

$$
S_{x x}(\omega)=\int_{-\infty}^{\infty} \gamma(\tau) e^{-i \omega \tau} d \tau=\hat{\gamma}(\omega)
$$

\section{System}

The schematic diagram of the testing system is presented in Fig.3 and the external view of the system is shown in Fig.4. Tungsten halogen lamp is employed as the light source, which generates the light of $2856 \mathrm{~K}$. The intensity of incident light will be reduced by optical filters and apertures. The MCPPMT detects incident photons placed on the support frame in the opaque chamber that provides a dark environment. The box is fabricated with soft iron, which improves magnetic shielding efficiency and avoid the influence of outer magnetic field. The anode and cathode current will be measured by corresponding galvanometer. The IPC samples anode and cathode current to calculate the parameters which are processed in software module.

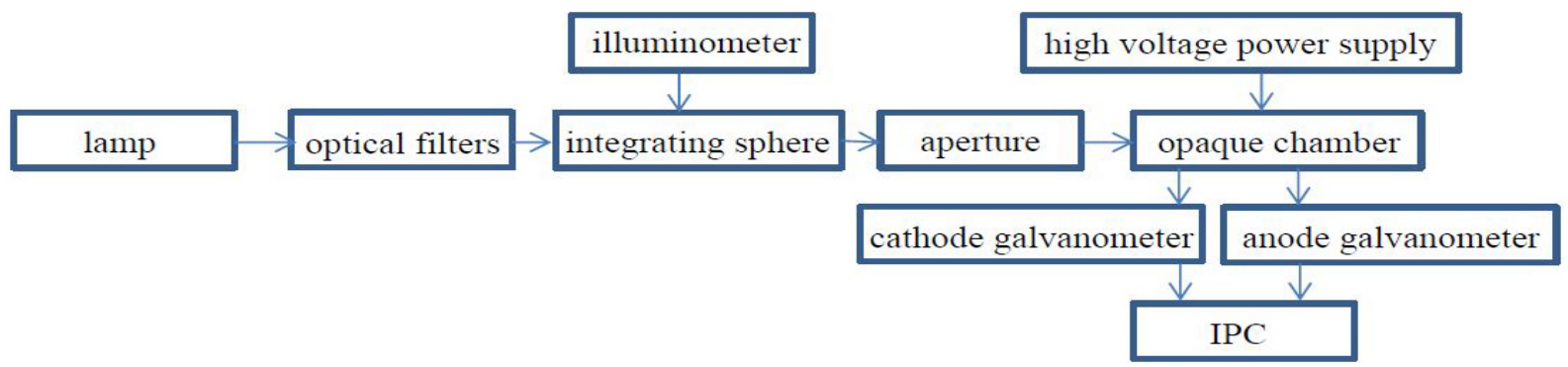

Fig. 3: The schematic diagram of the testing system 


\section{Analysis of Static Performance}

Power Spectrum. One set of the double MCPs is tested for the static performance evaluation. We change the applied voltages of two MCPs and sample the anode output current to analyze its noise power spectrum. The voltage between cathode and focusing electrode is $100 \mathrm{~V}$, which is the same with the voltage between anode and MCP2. The sampling frequency of IPC reaches $10,000 \mathrm{~Hz}$, and 100,000 data are sampled under every voltage. The power spectral density of anode current with incident light at $410 \mathrm{~nm}$ wavelength is presented in Fig.5, which has eliminated the power line interference.

In Fig.5, the power spectrum falls off steadily under $30 \mathrm{~Hz}$, which exhibits the property of $1 / \mathrm{f}$ noise, whereas the higher frequencies are overshadowed by low-pass filter in anode galvanometer. If the anode output current is kept low, thermal noise that is presented as the jitter at high frequencies on the power spectrum will be the predominant effect[7]. With the increasing of MCP's voltage, the noise power spectral density is boosted.

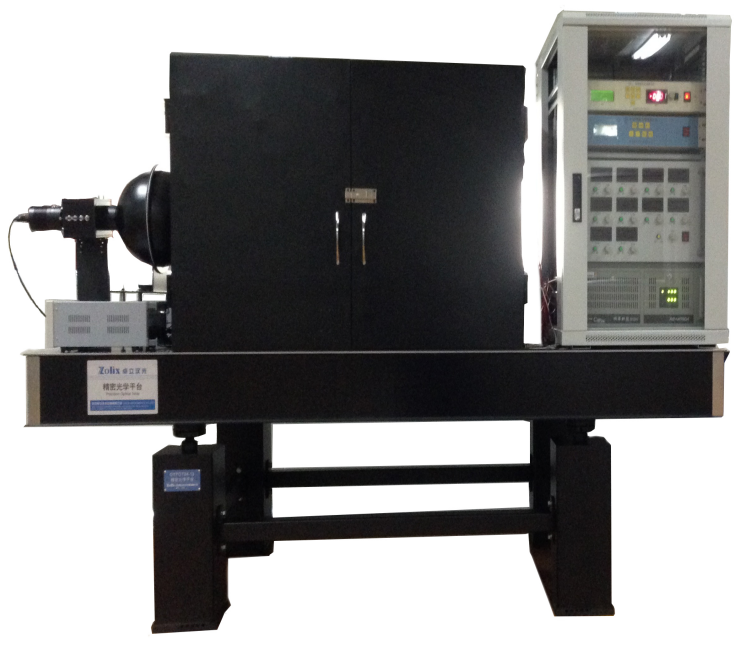

Fig. 4: The external view of the system

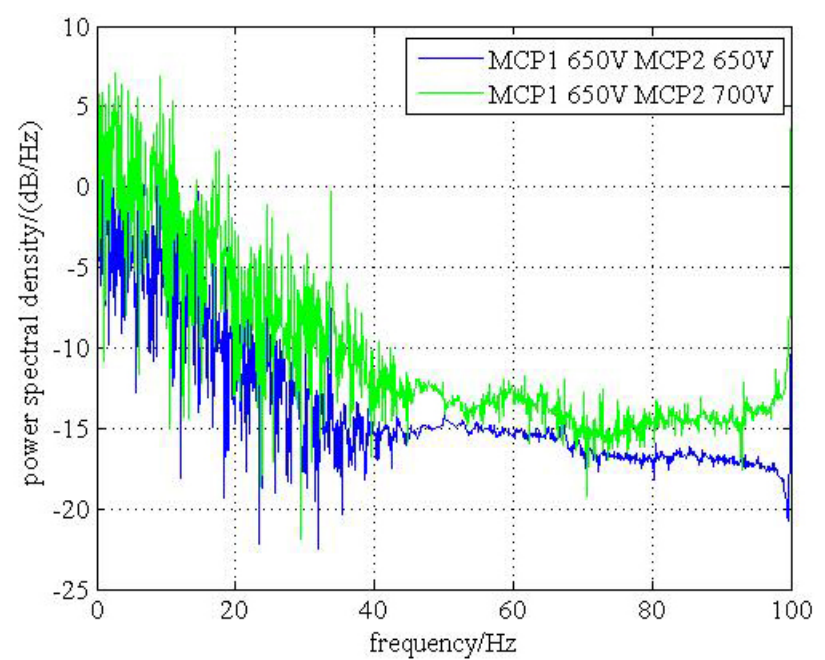

Fig. 5: The power spectral density of $410 \mathrm{~nm}$ incident light

Cathode Parameters. The cathode parameters are listed separately in Table 1 and Table 2. When the cathode parameters are tested, no voltages are applied to the studied MCPs. The photoelectrons collected by the focusing electrode are directly measured by the cathode galvanometer. Through the system, we can get the cathode parameters in full spectrum and $410 \mathrm{~nm}$ wavelength.

Table 1: The cathode parameters in full spectrum

\begin{tabular}{|c|c|c|c|}
\hline incident light & cathode current(nA) & cathode sensitivity $(\mu \mathrm{A} / \mathrm{lm})$ & luminous flux $(\mu l m)$ \\
\hline attenuated of $10^{2}$ & 0.847 & 41.26 & 20.5483 \\
\hline
\end{tabular}

Table 2: The cathode parameters at $410 \mathrm{~nm}$ wavelength

\begin{tabular}{|c|c|c|c|c|}
\hline incident light & cathode current(nA) & $\begin{array}{c}\text { cathode spectrum } \\
\text { sensitivity(mA/W) }\end{array}$ & quantum efficiency(\%) & radiant flux(nW) \\
\hline $410 \mathrm{~nm}$ & 3.400 & 80.12 & 24.21 & 42.52 \\
\hline
\end{tabular}

Anode Parameters. The anode parameters are listed separately in Table 3 and Table 4 . When the parameters in full spectrum are measured, the intensity of incident light is reduced $10^{4}$ level by employing appropriate neutral optical attenuator. When the parameters at $410 \mathrm{~nm}$ wavelength are measured, the neutral optical attenuator of $10^{4}$ and $410 \mathrm{~nm}$ optical filter are both applied to obtain monochromatic light incidence .

As shown in Table 3 and Table 4, the anode parameters rise with the increasing of MCPs' voltages. The testing system measures the anode current precisely and computes other corresponding parameters which are compatible with the typical properties of MCP-PMT. 
Table 3: The anode parameters in full spectrum

\begin{tabular}{|c|c|c|c|c|c|}
\hline MCP1(V) & MCP2(V) & dark current $(\mu A)$ & anode current $(\mu A)$ & anode sensitivity(A/lm) & gain \\
\hline 750 & 750 & 1.521 & 16.634 & 1.280 & $3.101 \times 10^{4}$ \\
\hline 750 & 700 & 1.650 & 14.623 & 1.099 & $2.663 \times 10^{4}$ \\
\hline 650 & 700 & 0.973 & 11.235 & 0.864 & $2.095 \times 10^{4}$ \\
\hline 650 & 650 & 0.714 & 9.697 & 0.746 & $1.808 \times 10^{4}$ \\
\hline
\end{tabular}

Table 4: The anode parameters at 410nm wavelength

\begin{tabular}{|c|c|c|c|}
\hline MCP1(V) & MCP2(V) & anode spectrum sensitivity(A/W) & anode current $(\mu A)$ \\
\hline 750 & 750 & $3.714 \times 10^{5}$ & 1.579 \\
\hline 750 & 700 & $1.244 \times 10^{5}$ & 0.529 \\
\hline 650 & 700 & $7.949 \times 10^{4}$ & 0.338 \\
\hline 650 & 650 & $5.715 \times 10^{4}$ & 0.243 \\
\hline
\end{tabular}

\section{Conclusions}

According to the testing results, the system provides a useful method to approach the static performance of MCP-PMT, such as gain, anode current, cathode current, anode sensitivity, cathode sensitivity and quantum efficiency. These properties can be measured precisely in different MCPs' voltages, which reflects the reliability of the system. In addition, we can distinguish the $1 / \mathrm{f}$ noise in the power spectrum of anode current and confirm the higher frequencies are restrained by the low-pass filter in anode galvanometer. In the present work, the system is qualified to test the static performance of MCP-PMT.

\section{Acknowledgement}

This work was supported by grants from the National Undergraduate Innovative Test Program,P.R.China.

\section{References}

[1] M.C.Sanchez, et, al., Using Large Area Microchannel Plate Photodetectors in the Next Generation Water Cherenkov Neutrino Detectors. Nuclear Physics B-Proceedings Supplements, 229-232 (2012) 525.

[2] A.Yu.Barnyakov, et, al., Photomultiplier tubes with three MCPs. Nuclear Instruments and Methods in Physics Research A, 598(2009), 160-162.

[3] Yifang Wang, et, al., Jinshou Tian. A new design of large area MCP-PMT for the next generation neutrino experiment. Nuclear Instruments and Methods in Physics Research A, 695(2012), 113 117.

[4] Ruishan Bai. Application and test on the characteristic of two-piece cascaded MCP. Nanjing: Nanjing University of Science and Technology, 2014: 7-13.

[5] Guihua Wang. Optoelectronic Devices. Beijing: National Defense Industry Press, 2014: 75-80.

[6] Information on http:// en.wikipedia.org.

[7] Yunsheng Qian, Benkang Chang, Moying Tong, Lei Liu. Frequency Spectrum Measurement of Noise of Image Intensifiers. ACTA OPTICA SINICA, 2003, 23(1): 67-70. 